\title{
ViPS: Visual Processing System for Medical Imaging
}

\author{
Tassadaq Hussain ${ }^{1}$, Oscar Palomar ${ }^{2}$, Adrian Cristal ${ }^{2}$, Eduard Ayguadé \\ ${ }^{1}$ Riphah International University Islamabad, Pakistan ${ }^{2}$ Computer Sciences, Barcelona Supercomputing Center, Barcelona, Spain \\ Email: \{tassaduq.hussain@ riphah.edu.pk\}
}

\begin{abstract}
Imaging has become an indispensable tool in modern medicine. Various powerful and expensive platforms to study medical imaging applications appear in recent years. In this article, we design and propose a Visual Processing System (ViPS) that processes medical imaging applications efficiently. ViPS provides a user-friendly programming environment and highperformance architecture to perform image analysis, features extraction and object recognition for complex real-time images or videos. The data structure of image or video is described in the program memory using pattern descriptors; ViPS uses specialized 3D memory structure to handle complex images or videos and processes them on microprocessors or application specific hardware accelerators. The proposed system is highly reliable in terms of cost, performance, and power. ViPS based system is implemented and tested on a Xilinx Virtex-7 FPGA VC707 Evaluation Kit. The performance of ViPS is compared with the Intel i7 multi-core, GPU Jetson TK1 Embedded Development Kit with 192 CUDA cores based graphic systems. When compared with the Intel and GPU-based systems, the results show that ViPS performs real-time video reconstruction at $2 x$ and $1.45 x$ of higher frame rate, achieves $14.6 x$ to $4.8 x$ of speedup while executing different image processing applications and $20.3 \%$ and $12.6 \%$ of speedup for video processing algorithms respectively.
\end{abstract}

\section{INTRODUCTION}

Graphics software programs are growing and are desirable on a big spectrum ranging from medical science to gaming technology because they can generate realistic images and enable graphics effects for user interfaces that give different viewpoints and visual clues. Different software engines [1] [2] [3] are introduced which provide an efficient means of reuse between a set of related products. Those software solutions do provide flexibility and re-programmability, but graphics performance is limited by the computation power of graphics devices.

Graphics systems are now being used in medical to diagnose and manage the physical form of patients. As the biomedical industry tries to lower the patient cost and achieves earlier disease prediction, the medical imaging equipment takes on an increasingly critical role in health care. To meet these industry goals, the bio-medical industry is pushing towards high-performance computing designs. As the performance of these devices grows, application specific and high-performance hardware are required to run complicated/complex applications.

A number of High Performance Computing (HPC) graphics engines e.g ATI [4] and nVidia [5] are now available in the market. A major drawback of these architectures is the lack of programming models for the medical imaging applications.

The research leading to these results has received funding from the Riphah International University Islamabad Pakistan.
These architectures use their knowledge of the personal computer market and provide a generic programming model for the HPC applications. These architectures do not fulfill demands of medical scientists for solving the medical imaging problem. Therefore, the medical imaging industry needs an architecture that not only gives high performance but also provides a programming model that facilitates the medical scientist to write their application without going into detail of hardware design.

In this work, we intend to develop a low-power, lowcost, easy to use and high-performance graphics architecture called Visual Processing System (ViPS). ViPS provides a high performance FPGA-based design which takes complex image/video data from medical imaging interfaces or stored in the memory, manages them in an on-chip Specialized Memory and process them using specialized hardware accelerators or multi-core system. The ViPS programming model aims to remove the programming effort of manually arranging data transfer requests, memory management, input/output peripheral management and meet the performance requirements of the imaging applications. The approach reduces the application processing time, gives promising interconnection approach for multiple imaging peripherals with the potential to exploit parallelism while copying the memory/network latencies and balances the workload. ViPS bus scheduler provides low-cost and simple control characteristics that arranges multiple imaging peripherals requests and communicates with integrated processing units. We integrated dedicated hardware accelerators in the design as they have a low footprint and low power consumption and gives high-performance computation. ViPS supports multi-peripherals (camera, display) and processor core without the support of the master cores and operating system (OS). The integration of ViPS with peripherals facilitates the graphics system to overcome wire (interconnection) and memory read/write delays and improves the performance of application kernels by arranging complex on-chip data transfers. While comparing results with the Intel and GPUbased systems, it show that ViPS performs real-time video reconstruction at $2 \mathrm{x}$ and $1.45 \mathrm{x}$ of higher frame rate, achieves $14.6 \mathrm{x}$ to $4.8 \mathrm{x}$ of speedup while executing different image processing applications and $20.3 \%$ and $12.6 \%$ of speedup for video processing

The rest of this paper is organized as follows: Section III describes ViPS system. Section IV presents the results. Finally, Section II discusses the related work and Section V provides the conclusions. 


\section{RELATED WORK}

Bakalash et al. [6] proposed MediCube system for 3D medical imaging. The system supports the reconstruction, manipulation, analysis, and display of 3D volumetric medical images. The system is based on general-purpose voxel-based Cube architecture, which employs parallel memory and parallel processing to support real-time manipulation and display of voxel imagery. The ViPS handles 3D medical imaging using specialized scratchpad memory and uses reconfigurable application specific hardware accelerators.

Specialized DSP based systems like the Bluetechnix [7] Blackn camera boards provide superior image processing abilities at the expense of power, price, and complexity. Lee et al. [8] offered SONY digital signal processor (DSP) based advanced video camera system which has high automatic focus (AF), automatic exposure (AE), and automatic whitebalance (AWB) control. The ViPS system offers low cost and low power architecture with the support of image processing abilities. Jinghong et al. [9] proposed an Image processing system structure based on DSP and FPGA. The system uses DSP as advanced image processing unit and FPGA as a logic unit for image sampling and display. The developed system can take image, display image and make some image processing operations that include geometry transform, orthographic transform, operations based on pixels, image compression and color space conversion. ViPS uses application specific hardware accelerators for high performance applications and to take high-speed data from sensors. A 32-bit RISC core is integrated with the design for programmability, moderate performance and low cost systems.

Tassadaq et al. proposed Programmable Graphics Controller [10] [11] for low cost and low power graphics system. The system takes 2 dimensional images to process applications. ViPS uses specialized memory which helps architecture to execute 3 dimensional images for medical applications. The ViPS architecture provides easy to use programming environment for the applications.

\section{ViPS GRAPhics System SPECIFICATION}

Architectural investigation for visual processing system ranges from high-level system architecture to analog and circuit-level design. ViPS architecture covers the reuse of processing elements, data parallelism and the network architecture.

In this section, we describe the specification of ViPS system and design its architecture. The section is further categorized into five subsections: Overview of ViPS, the Network Unit, Memory Unit, Processing Units and Programming Model.

\section{A. Overview of ViPS}

ViPS architecture is pipelined from the sensor chip over the wire to the processing chip, detailing data flow and onboard data storage. ViPS inner architecture is shown in Figure 1, which displays the interconnection of the processing units and memory. The system uses combined hardware/software solution that includes hardware accelerators, and an RISC

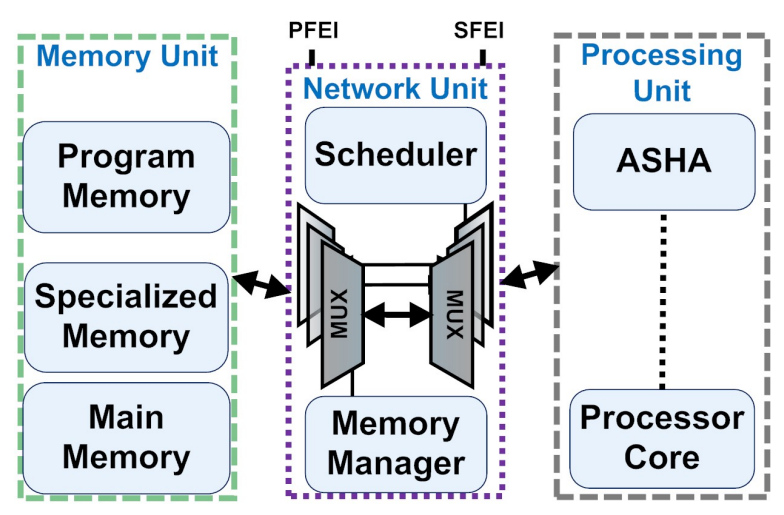

Fig. 1. ViPS : Internal Structure

processor core. The camera and display are controlled by custom Application Specific Hardware Accelerators (ASHA). The Specialized Memory holds the complex image data for image/video processing and efficiently accesses, reuses and feeds data to the Processing Unit. To store high resolution/dencity images the Main Memory is integrated. The Program Memory is used to hold application program description and data transfer information. Depending upon the data transfer, the Memory Manager takes single or multiple instructions from Program Memory and schedules the data movement. The ViPS Scheduler handles the concurrent bus request by different analog peripherals (e.g. camera and display) and rearranges multiple data access requests and arbitrates data transfer without creating on-chip bus contention.

\section{B. Memory Unit}

The ViPS memory is organized into three sections: the Program Memory, the Specialized Memory, and the Main Memory.

1) Program Memory: The Program Memory holds descriptors [12], [13] that define the data movement between the processing unit and the memory unit. The descriptors allow the programmer to describe the shape and size of images and its location in memory. A single descriptor is represented by parameters called command, source address, destination address, stream, and stride. A command specifies the operation to be performed. The address parameters specify the source and destination locations. Stream defines the number of pixels to be transferred. Stride indicates the distance between two consecutive memory addresses of a stream. $\mathrm{C} / \mathrm{C}++$ function calls are provided to define a complex image structure in software.

2) Specialized Memory: The ViPS Specialized Memory [14] (SM) is directly connected to the Processing Unit and provides single cycle data access. Like a cache, the $S M$ temporarily holds data to speed up later accesses. Unlike a cache, data is deliberately placed in the $S M$ at a known location, rather than automatically cached according to a fixed hardware policy. The on-chip encapsulation of $S M$ with Processing Unit allows applications to access data without the additional delay of on-chip data management. Depending 


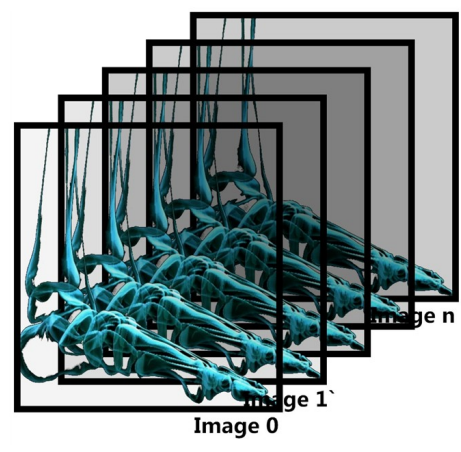

(a)

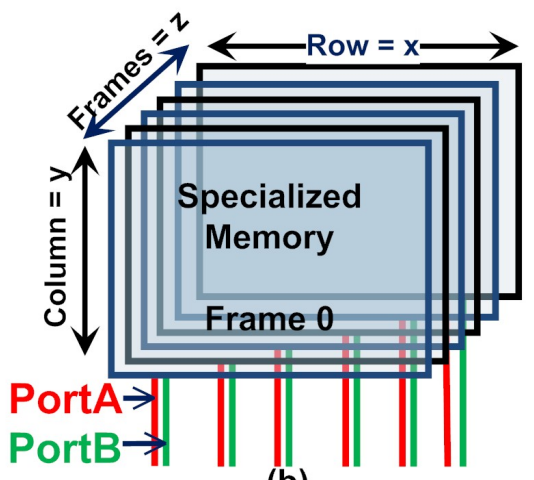

(b)

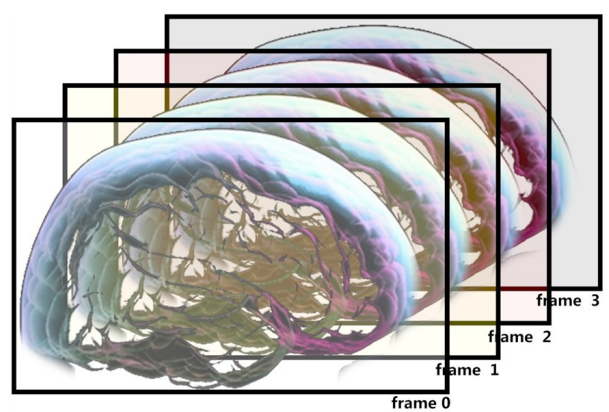

(c)

Fig. 2. (a) 3D Medical Image of Human Foot (b) ViPS Specialized Memory Architecture (c) 3D Image Placed in Specialized Memory

upon the available block RAMs, the $S M$ can be organized into multiple banks. Each bank has two ports (PortA \& PortB), which allows the Processing Unit to perform parallel reads and writes data. To exploit parallelism better, the banks of $S M$ are organized physically into a multi-dimensional (1D/2D/3D) architecture to map the kernel access pattern on the $S M$.

For example, a generic 3D image structure is shown in Figure 2(a). The $S M$ shown in Figure 2(b) uses multiple banks to accommodate 3D image. An example of 3D image placed in $S M$ is shown in Figure 2(c). Each bank handles a single image and its read/write operation is independent of other banks and can be performed in parallel.

In our current evaluation on Xilinx Virtex-7, the $S M$ has 8 banks and each bank holds 3 KPixel image. Each bank uses multiple BRAM, which is controlled by a separate BRAM controller and has a different base address. Single or two dimension data sets are placed in a single bank and can use single or multiple BRAM/s. ViPS accesses and places data in tiles if the data set is too larger than the $S M$ structure.

3) Main Memory: The slowest type of memory in the ViPS architecture is Main Memory and is accessible by the whole system. The Main Memory has SDRAM, SD/SDHC memories, etc. interfaces to read/write data.

\section{Network Unit}

The Network Unit transfers data from external graphic components such as camera $\&$ display sensors to the processing core. The data width of Network Unit is a significant factor; there are two types of interfaces that ViPS system supports: the Parallel Front-End Interface (PFEI) and the Serial Front-End Interface (SFEI). The RGB Raw Data and ITU-R 656 (YUV) are $P F E I$ and are available nearly in all graphic sensors and are used for low cost applications without integrated Image Signal Processor (ISP). The interface has a frame rate limitation for higher resolutions and has Electro Magnetic Interference issues at high pixel clock. Most of the textitSFEI are based upon LVDS (Low Voltage Differential Signaling) or subLVDS. LVDS make use of low-voltage differential signals and drives point-to-point and multi-drop by using low-voltage, low-power, and differential technology. The most attractive features of $L V D S$ are high signaling rate, low power consumption, and electromagnetic compatibility.

The ViPS Network Unit uses Scheduler and Memory Manager, to manage the processing units and memory units. The ViPS Scheduler along with Memory Manager arrange requests are coming from single or multiple imaging peripherals. The Memory Manager holds the address and control information and plays a critical role in managing and allocating data for the application kernel.

The memory accesses are rearranged during compile-time, to fit in the minimum number of descriptors of the Program Memory. At run-time the Memory Manager executes these descriptor blocks in hardware without generating overhead such as the time spent handling request and grant signals between processor and memory, as well as an address generation delay. At run-time, the Memory Manager executes these descriptor blocks in hardware, typically overlapping address generation and memory requests with computation in the processing unit. The Memory Manager manages the access patterns of application kernels with complex image data layouts. It keeps the information of the data currently stored in the $S M$ and reuses data when possible.

\section{Processing Unit}

ViPS supports two types of cores: the Application Specific Hardware Accelerator and the RISC Core.

1) Application Specific Hardware Accelerators: Application Specific Hardware Accelerators (ASHA) are used in the design for the imaging peripherals. A camera ASHA grabs raw data from the image sensor, processes it and transfers it to the system via the Network Unit. The primary function blocks of a camera ASHA are Camera Interface Front-End, Image Signal Processor, Color processing, Scaling, Compression, and Bus controller. To display image data on LCD panel, a display ASHA is used. It supports LCD 16bpp up to 24bpp colors and user defined resolution from VGA to QSXGA. Programming is done by register read/write transactions using a slave interface.

2) Processor Core: A low power and light weight 32bit RISC processor core is used to provide programmability, flexibility and software data processing. ViPS is using a 
software interface API that can be used to correct design errors, update the system to a new graphic standard and to add more features to the graphics system. The proposed processor core has 32-bit data bus, 32-general purpose registers, custom instruction set, non-pipelined Load/Store access, hardwired control unit, 64KByte address space, total 16-interrupts and memory mapped I/Os.

\section{E. Programming Model}

When using ViPS programming model, the programmer does not need to worry about the hardware related programming and configuration constraints. By using ViPS, the memory operations are shaped into patterns and are scheduled in parallel with the processing unit. ViPS supports complex irregular, strider 1D, 2D, 3D and automated blocking for image/video access operations to transfer data between, Network Unit, Local and Main memories. The Processing Unit communicate with ViPS through a group of commands, controls, status and data registers and signals. Table I shows function calls to programm the current ViPS architecture.

\section{Results And Discussion}

In this section, we analyze the results of different experiments conducted on ViPS. In order to evaluate the performance of ViPS, the results are compared with Intel i7-2670QM quadcore (2.2 GHz, 6MB Cache) based laptop and the GPU Jetson TK1 board having a quad-core ARM Cortex-A15 processor and 192 CUDA cores. The architectures are connected with CMOS and Ultrasonic imaging sensors. The experiments are classified into three subsections: Real-time Image Reconstruction, Image Processing and Video Processing.

\section{A. Real-time Image Reconstruction}

For complex imaging algorithms, the computational complexity of image reconstruction has increased dramatically. Therefore, high-speed image reconstruction is required which is even more critical in real-time imaging applications, such as online adaptive therapy. Ultra-fast image reconstruction could also allow the clinician to adjust reconstruction parameters interactively and optimize the noise/spatial resolution trade-off. A multi-camera graphics system can be used for 3D-graphics using geometric transformation and projection plane [15]. In this section, two THDB-D5M image sensors are used that generate two separate, simultaneous video streams and apply Alpha blending application that evaluate the performance of the system. Each camera is operating at VGA color resolution. The video of dual image sensors is combined into a single

TABLE I

C/C++ Device Drivers to Program/Operate ViPS

\begin{tabular}{|l|l|}
\hline API Function & Description \\
\hline READ_IMAGE, WRITE_IMAGE, & Image Data Access \\
ViPSMEMCPY 1 & SPECIALIZEDMEMORY \\
SPECIALIZED_MEMORY, & indicates Local Memory buffer \\
DATA_SET, Priority) & DATA_SET indicates Main Memory data set \\
\hline 3D_EILTER ( & Specialized Data Transfer \\
SPECIALIZED_MEMORY, & SPECIALIZED_MEMORY indicates SM structure \\
DATA_SET, Kernel, Priority) & DATA_SET indicates Main Memory data set \\
& Kernel defines the type of filter \\
\hline
\end{tabular}

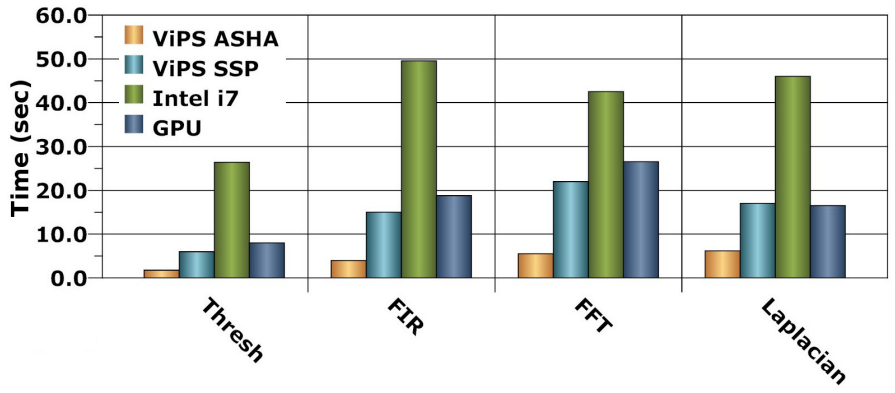

Fig. 3. Applications Executing Time: ViPS ASHA, ViPS SSP, Intel i7 and GPU

stream, processed by graphics core and then displayed. The key issue of the dual-camera system is receiving the images synchronously, in the right format and on the right bus. The graphic system sends the configuration data to both image sensors and ensures that they are properly configured and synchronized. Once both sensors are set up and synchronized, both sensors begin to transmit image data. The graphic system looks for the appropriate control characters, so it recognizes the start of the frame and start of a line for each sensor. ViPS performs it by looking for a control character and sequence of sensors commands. Alpha blending is applied to give a translucent effect to the incoming video stream. The application blends the color value of the consecutive pixels of image sensors of the same position. This blending is done according to the alpha value associated with the pixel. The alpha value represents the capacity of the given pixel. Results show that ViPS handles dual camera system and support system up to $50 \mathrm{fps}$. The Intel i7 and GPU based dual-camera graphics system supports videos up to 25 and 35 fps respectively. The ViPS on-chip scheduler update multicamera information in status register. This allows both cameras to synchronize without using extra clocks.

\section{B. Image Processing}

In this section, we execute Thresholding (Thresh), Finite Impulse Response (FIR), Fast Fourier Transform (FFT) and Laplacian Filter (Laplacian) application kernels on ViPS SSP and ViPS ASHA based systems. ViPS SSP and ViPS ASHA systems execute application kernels on soft scalar processor and application-specific hardware accelerators respectively. Figure 3 shows applications execution time. $\mathrm{X}$-axis presents applications name, and Y-axis displays execution time in seconds (lower is better). ViPS results are compared with the Intel 17 and GPU based system. The systems read one still image of QSXGA resolution from the camera sensor and write it to the Main Memory. The processor core reads the image, performs computation and then writes it back to the Main Memory. The results show that while executing Thresh, ViPS ASHA achieves 14.6x and 4.4x of speedups compare to the Intel i7 and GPU systems respectively. This application kernel requires single pixel element and very few operation. The FIR application has streaming data access 
pattern and performs multiplication and addition. ViPS ASHA achieve $12.3 \mathrm{x}$ and $4.7 \mathrm{x}$ of speedups respectively. The FFT application kernel reads a 1D block of data, perform complex computation and writes it back to the Main Memory. ViPS ASHA achieve $7.7 x$ and $4.8 x$ of speedups respectively. The Laplacian application kernel processes over a 2D block of data, ViPS ASHA make 7.4x and 2.6x of speedups respectively. ViPS places access patterns on Program Memory at program time and are programmed in such a way that few operations are required for generating addresses at run-time. The Intel i7 and GPU-based systems use multiple load/store, or DMA calls to access complex patterns. The speedups are possible because ViPS can manage data transfers with a single Descriptor. At run-time, ViPS takes Descriptor from Program Memory independently and manages them in the Specialized Memory, whereas the baseline systems are dependent on the processor core that manages on chip data, data transfer instructions and the Main Memory data. The stand-alone working operation of ViPS removes the overhead of processor/memory system request/grant delay.

\section{Video Processing}

In this section, we use real-time data from different sensors and applies video processing algorithms using CMOS image sensor. The systems apply Object Detection, Object Recognition, 3D-Stereo Filtering and Ultrasonic Image Reconstructing on real-time sensor data for 10 minutes shown in Figure 4. X-axis presents applications processed by ViPS, Intel i7, and GPU systems. ViPS system processes an application using ASHAs. Y-axis shows number of processed frames in 10 minutes by each system. The CMOS image sensor is programmed at $(640 \times 480)$ resolution and 30 (frame per second) ( fps) therefore maximum number of frames are 18000. Each bar presents the number of processed frames per 10 minutes (higher is better). If the application is complex, the system takes more time and skips some of the frames. We measure only those frames that are processed by the system. While executing Object Detection application, the results show that ViPS achieves $20.3 \%$, and $8.4 \%$ of speedups

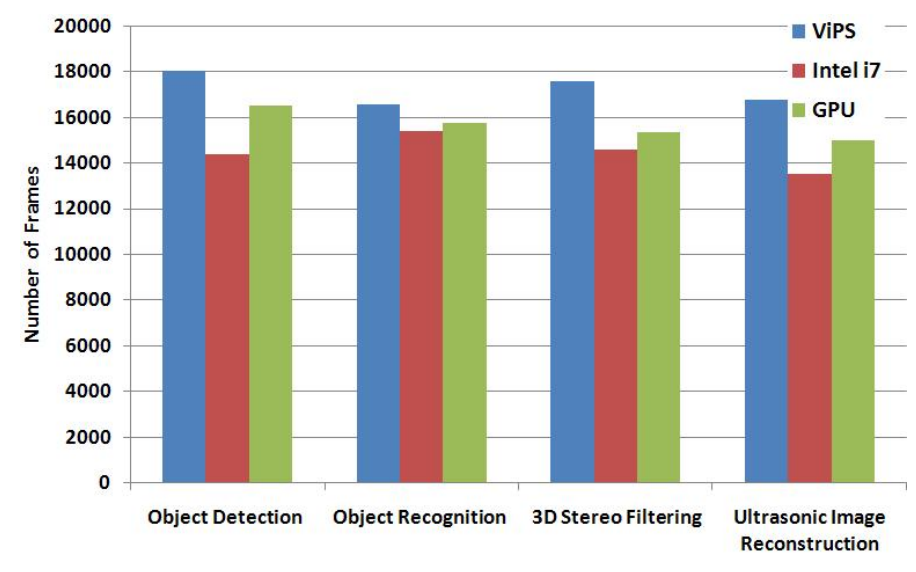

Fig. 4. Video Processed Frames: ViPS, Intel i7 and GPU compare to the Intel i7 and GPU systems respectively. This application takes 2D images directly from the image sensor. The Object Recognition application reads a 2D block of data and performs recognition algorithm. ViPS achieve $7.7 \%$ and $4.8 \%$ of speedups respectively. The $3 D$ Stereo Filtering application processes over a 3D block of data, ViPS reach $17.4 \%$ and $12.6 \%$ of speedups respectively. ViPS uses Specialized Memory to handle 3D stereo images and organize the graphic data access patterns in the form of descriptors which takes few operations for generating addresses at run-time. We also use the ultrasound sensor to create an image from sound and applies Ultrasonic Image Reconstruction using Bayesian Image Reconstruction [16]. The results show that ViPS achieve $19.4 \%$ and $10.6 \%$ of speedups respectively while applying Ultrasonic Image Reconstruction application over ultrasound sensor.

\section{CONCLUSION}

In this paper, we have suggested a Visual Processing System (ViPS) for medical imaging applications. The system takes high-resolution images and supports video at higher frame rate without the help of a processor. The ViPS system provides efficient data access from image sensors that eliminate the on-chip/off-chip bus delays for arranging and gathering data. In the future, we plan to provide more functions to support image/video processing applications.

\section{REFERENCES}

[1] OGRE: Object-Oriented Graphics Rendering Engine. http://www. ogre3d.org/.

[2] Irrlicht: An Open Source High Performance Realtime 3D Engine. http: //irrlicht.sourceforge.net/.

[3] Ali et al. Alzaabi. Tcct: A gui table comparison computer tool. In Emerging Trends in Computing, Informatics, Systems Sciences, and Engineering. Springer.

[4] ATI Technologies Inc. http://www.amd.com/.

[5] Visual computing technology from NVIDIA. http://www.nvidia.com/.

[6] Reuven Bakalash and Arie Kaufman. Medicube: A 3d medical imaging architecture. Computers \& Graphics, 13(2), 1989.

[7] Bluetechnix Black DSP. http://www.xbow.com.

[8] Lee, June-Sok and Jung, You-Young and Kim, Byung-Soo and Ko, SungJea. An advanced video camera system with robust AF, AE, and AWB control. IEEE Transactions on Consumer Electronics.

[9] Jinghong, Duan and Yaling, Deng and Kun, Liang. Development of image processing system based on DSP and FPGA. In Electronic Measurement and Instruments, 2007. ICEMI'07.

[10] Tassadaq Hussain, Oscar Palomar, Adrian Cristal, Osman Unsal, Eduard Ayguady, Mateo Valero and Amna Haider. Stand-alone Memory Controller for Graphics System. In The 10th International Symposium on Applied Reconfigurable Computing (ARC 2014). ACM, 2014.

[11] Tassadaq Hussain and Amna Haider. PGC: A Pattern-Based Graphics Controller. Int. J. Circuits and Architecture Design, 2014.

[12] Tassadaq Hussain, Miquel Pericas, Nacho Navarro and Eduard Ayguade. Reconfigurable Memory Controller with Programmable Pattern Support. HiPEAC Workshop on Reconfigurable Computing, Jan, 2011.

[13] Tassadaq Hussain, Muhammad Shafiq, Miquel Pericas, Nacho Navarro and Eduard Ayguade. PPMC: A Programmable Pattern based Memory Controller. In ARC 2012.

[14] Tassadaq Hussain, Oscar Palomar, Adrian Cristal, Osman Unsal, Eduard Ayguady and Mateo Valero. Advanced Pattern based Memory Controller for FPGA based HPC Applications. In International Conference on High Performance Computing \& Simulation, page 8. ACM, IEEE, 2014.

[15] Richard Hartley and Andrew Zisserman. Multiple view geometry in computer vision, volume 2. Cambridge Univ Press, 2000. 
[16] Jinyi Qi, Richard M Leahy, Simon R Cherry, Arion Chatziioannou, and Thomas H Farquhar. High-resolution 3d bayesian image reconstruction using the micropet small-animal scanner. Physics in medicine and biology, 43(4), 1998. 